\title{
Heat Capacity of Silver lodide. II. Theory
}

Cite as: J. Chem. Phys. 48, 2681 (1968); https://doi.org/10.1063/1.1669502

Submitted: 10 October 1967 . Published Online: 05 September 2003

C. M. Perrott, and N. H. Fletcher

\section{ARTICLES YOU MAY BE INTERESTED IN}

Heat Capacity of Silver lodide. I. Experiments on Annealed Samples

The Journal of Chemical Physics 48, 2143 (1968); https://doi.org/10.1063/1.1669404

Heat Capacity of Silver Iodide. III. An Order-Disorder Transition

The Journal of Chemical Physics 50, 2770 (1969); https://doi.org/10.1063/1.1671448

Heat Capacity of Silver Sulfide

The Journal of Chemical Physics 50, 2344 (1969); https://doi.org/10.1063/1.1671386

Meet the Next Generation of Quantum Analyzers And Join the Launch Event on November 17th

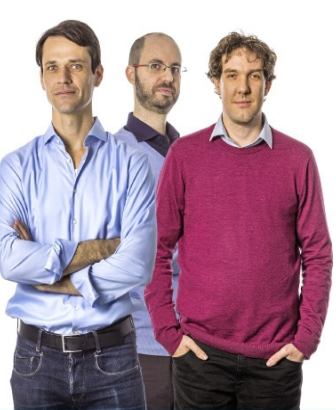

Register now

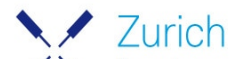
- Instruments 
synthesize the spectrum in Fig. 6 and the spectrum below the horizontal line in Fig. 5 relative to $\Delta \omega^{s}+D^{s}$. The energy separations in $\mathrm{cm}^{-1}$ are based upon our own measurements of absolute absorption intensities of $\mathrm{CuPC}$ in 1-chloronaphthalene, $f=0.20, M^{2}=1.2 \AA^{2}$.

Size and shape effects do not alter the quantitative results significantly nor do they affect the assignments of the exciton components.

\section{Mixtures}

In the mixtures of $\alpha$ and $\beta$ crystals the positions of the absorption maxima would be expected to shift with composition according to the expression $v_{\mathrm{B}}=a+b m_{\mathrm{A}}+$ $c m_{\mathrm{A}}{ }^{2}$ obtained by rearrangement of Salomon's ${ }^{13} \mathrm{Eq}$. (5). In this equation $m_{\mathrm{A}}$ is the mole fraction of $\mathrm{A}$ molecules randomly distributed in an isomorphous host crystal of $\mathrm{B}$ molecules, and the constants $a, b$, and $c$ depend upon the separate transition energies in $A$ and $\mathrm{B}$ and the $\mathrm{A}-\mathrm{A}, \mathrm{B}-\mathrm{B}, \mathrm{A}-\mathrm{B}$ interaction energies.

In order to test the quadratic dependence of peak position upon concentration predicted by this theory, a higher precision is required than the $\pm 0.5 \%$ obtained in this work. For the most part, the spectral shifts do not follow a regular pattern. However, in Table I within experimental error an approximately quadratic dependence is evident for the near $14700-\mathrm{cm}^{-1}$ band in $\alpha$ crystals of $\mathrm{CoPC}$ and NiPC mixtures with $\mathrm{CuPC}$ and for the $\sim 16000-\mathrm{cm}^{-1}$ band of $\mathrm{H}_{2} \mathrm{PC}$ and $\mathrm{CoPC}$ with $\mathrm{CuPC}$. The $\alpha-\mathrm{ZnPc}-\mathrm{CuPC}$ mixtures show a nearly linear dependence. In Table II the $\beta$-ZnPCCuPC mixtures also show a nearly linear dependence within the limits of our precision.

\section{ACKNOWLEDGMENTS}

We wish to extend our thanks to Mrs. Doris Stone for computer calculations of the lattice sums at Frankford Arsenal. Also, we hereby express our gratitude to Dr. Assour and Dr. Harrison at RCA Laboratories for the pure samples and much helpful advice. We are also grateful to Professor R. E. Salomon of Temple University, and to Henry Katz and others at the University of Pennsylvania for many helpful discussions.

THE JOURNAL OF CHEMICAL PHYSICS

VOLUME 48 , N UM B E R 6

15 MAR CH 1968

\title{
Heat Capacity of Silver Iodide. II. Theory
}

\author{
C. M. Perrott and N. H. Fletcher \\ Department of Physics, University of New England, Armidale, New South Wales, Australia
}

(Received 10 October 1967)

\begin{abstract}
Experiment has shown that the specific heat of stoichiometric $\alpha$-AgI is abnormally large, $C_{v}$ ranging from $18 \mathrm{cal} / \mathrm{mole} \cdot{ }^{\circ} \mathrm{C}$ at $150^{\circ} \mathrm{C}$ to $26 \mathrm{cal} / \mathrm{mole} \cdot{ }^{\circ} \mathrm{C}$ at $400^{\circ} \mathrm{C}$. This result is explained as an order-disorder transition among silver ions and silver ion vacancies on six sublattices in the structure. Because of the complex nature of the lattice sites, each of which consists of a cluster of seven nonequivalent potential wells, there is also a major anomalous contribution to the specific heat from the vibrational partition function. In addition there may be a small contribution from a coupled disordering of iodide ions. The behavior of nonstoichiometric $\alpha$-AgI, which has $C_{v} \approx 12 \mathrm{cal} / \mathrm{mole} \cdot{ }^{\circ} \mathrm{C}$, is attributed to essentially complete disordering above the phase transition at $150^{\circ} \mathrm{C}$ together with a modification of the vibrational potentials.
\end{abstract}

In the first paper of this series, ${ }^{1}$ to be referred to as I, an experimental study was made of the heat capacity of annealed silver iodide in the temperature range $50^{\circ}-400^{\circ} \mathrm{C}$. Below the phase change from the hexagonal $\beta$ form to the cubic $\alpha$ form at about $150^{\circ} \mathrm{C}$, the specific heat was close to normal $\left(C_{v} \approx 15 \mathrm{cal} / \mathrm{mole} \cdot{ }^{\circ} \mathrm{C}\right)$. For stoichiometric material, however, the heat capacity of the $\alpha$ form was anomalous, rising from $18 \mathrm{cal} / \mathrm{mole} \cdot{ }^{\circ} \mathrm{C}$ just above the transition to $26 \mathrm{cal} / \mathrm{mole} \cdot{ }^{\circ} \mathrm{C}$ at $400^{\circ} \mathrm{C}$. For silver iodide which was nonstoichiometric by as little as $1 \mathrm{~mole} \%$ in either direction, the specific heat of the $\alpha$ form was normal, having a value of $12 \mathrm{cal} / \mathrm{mole}$. ${ }^{\circ} \mathrm{C}$ from the transition up to at least $400^{\circ} \mathrm{C}$. The experimental data is summarized in Fig. 1.

${ }^{1}$ C. M. Perrott and N. H. Fletcher, J. Chęm. Phys. 48, 2143 (1968).
In the present paper we develop a theoretical model for the thermal behavior of stoichiometric $\alpha$-AgI, based upon the unusual crystal structure of this material, and show that it is able to account for the anomalously high observed specific heat. No detailed treatment of the way in which small deviations from stoichiometry remove this anomaly has yet proved possible but a plausible physical mechanism is suggested.

\section{PHYSICAL MODEL}

The crystal structure of $\alpha$-AgI has been examined by Strock, ${ }^{2}$ Hoshino, ${ }^{3}$ and others, using $x$-ray diffraction

\footnotetext{
${ }^{2}$ L. W. Strock, Z.'Physik.'Chem. B25, 441 (1934); B31, 132 (1936).

${ }^{3}$ S. Hoshino, J. Phys. Soc. Japan 12, 315 (1957).
} 


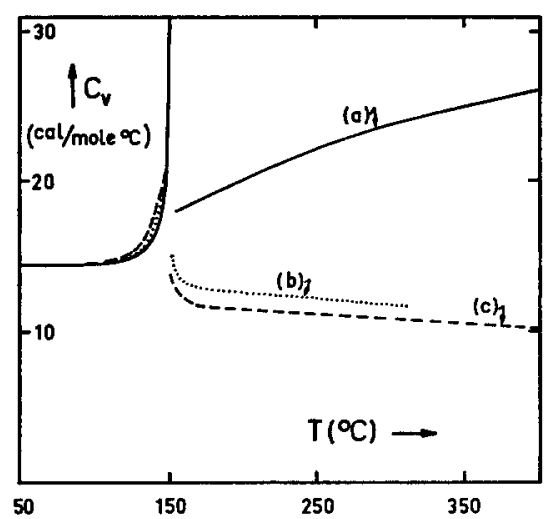

Fig. 1. Heat capacity of silver iodide for (a) stoichiometric material and for material of approximate composition, (b) $\mathrm{AgI}_{1 \cdot 01}$, and (c) $\mathrm{Ag}_{1 \cdot 01} \mathrm{I}$.

powder techniques. It has not yet been possible to prepare single crystals of the $\alpha$ form, either by heating crystals of the hexagonal $\beta$ form through the transition or by growth from the melt, which is chemically unstable. The generally accepted structure is one in which the iodide ions form a body-centered cubic structure while the silver ions are distributed more or less statistically over a large number of sites of roughly equivalent energy.

The sites available to the silver ions belong to the space group $I m 3 m$ and are labelled (b), (d), or (h) according to their symmetry. There are, in all, 42 positions for the two silver ions in the unit cell: six (b) sites, 12 (d) sites, and 24 (h) sites (Fig. 2). In the model proposed by Strock, the (b), (d), and (h) sites are taken as having equal occupation probabilities. Hoshino, on the other hand, showed that a model in which only (d) sites were occupied gave good agreement with the observed intensity of diffuse scattering. From this, we see that the assumption of different occupation probabilities for the different types of site has very little effect on the predicted diffuse scattering intensity. Although relative intensities of Debye lines may also be explained with this model, the absolute intensities cannot. It is probable that we could find an intermediate structure between these rather different models, which is capable of explaining the $\mathrm{x}$-ray data.

Such an intermediate model would predict a degree of disorder of the distribution of silver ions over the possible sites, which becomes larger with increasing temperature, as indicated by the observed increase in specific heat. Further evidence in support of an intermediate model is provided by Hoshino's observation that both silver and iodide ions have an anomalously large statistical displacement from their mean positions. The rms ionic displacement is the same for both species and increases from approximately $0.2 \AA$ at $160^{\circ} \mathrm{C}$ to approximately $0.6 \AA$ at $500^{\circ} \mathrm{C}$, this latter value being comparable with the nearest neighbor separation of silver sites, about $0.9 \AA$. At high temperatures, the ionic displacement is too large to be attributed to thermal vibration alone and must therefore be associated in some way with the disorder of the structure.

When we consider the magnitude of the lattice parameter ${ }^{3-5}\left(5.06 \AA\right.$ at $150^{\circ} \mathrm{C}$ and $5.14 \AA$ at $\left.500^{\circ} \mathrm{C}\right)$ in relation to the ionic radii ${ }^{6}$ ( $2.16 \AA$ for $\mathrm{I}^{-}$and $1.26 \AA$ for $\mathrm{Ag}^{+}$), we see that the two iodide ions nearest to an occupied (b) site, for example, must be displaced from their mean positions. If the disorder of silver ions increases with temperature, the rms displacement of the iodide ions will increase in amplitude, as observed.

The model which we propose for the thermal behavior of stoichiometric AgI is as follows. In a crystal containing $N$ silver ions and $N$ iodide ions, there are $3 N$ crystallographically equivalent sites of type (b) for silver ions. Each (b) site is surrounded by a cluster of (h) and (d) sites, of which its share is four (h) and two (d) sites. Each such cluster is then crystallographically equivalent. We are thus faced with the problem of distributing $N$ silver ions and $2 N$ silver ion vacancies over a total of $3 N$ equivalent sites, each site being not a simple potential well but a more complex well representing the cluster. Because of the relatively tight packing of the structure, this distribution cannot be made on an independent particle basis. In fact, before a silver ion can be placed on any site it is necessary to displace one or both of its neighboring iodide ions from their perfect lattice positions and this displacement will affect the potentials of all neighboring sites. The ordering or disordering of the silver ions and vacancies upon the sites of their lattice is thus expected to be a cooperative phenomenon. To a first approximation the treatment of this disordering process is the same as that for a simple $A_{2}$ alloy, where $A$ now represents a silver ion and $B$ a vacancy. The complicated nature of the clusters which make up the sites on the lattice is involved only through their

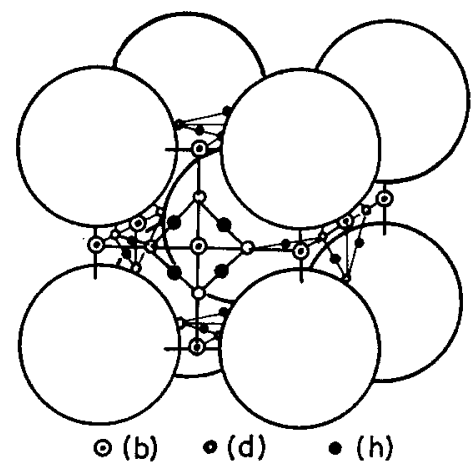

FIG. 2. The crystal structure of $\alpha$-AgI. Large spheres represent iodide ions and the small symbols show the clusters of sites available for silver ions.

${ }^{4}$ K. H. Lieser, Z. Physik. Chem. (Frankfurt) 5, 125 (1955),

5 B. R. Lawn, Acta Cryst. 17, 1341 (1964).

${ }^{\circ} \mathrm{L}$. Pauling, The Nature of the Chemical Bond (Oxford University Press, London, 1960), 3rd ed., p. 514. 
effect upon the vibrational partition function of each ion.

There is, however, a further complication when the lattice of iodide ions is taken into account. These have so far been treated as forming an ideal body-centered structure but suffering small displacements in order to accommodate the silver ions. The arrangement of the iodide ions among the displaced positions may itself be an order-disorder problem, the coupling from one site to another being provided by the distribution of the silver ions.

In the following sections we shall express these ideas in formal mathematical terms and derive an expression for the variation of disorder with temperature, from which the heat capacity can be deduced.

\section{MATHEMATICAL TREATMENT}

\section{An Outline of the Theory of Order-Disorder Phenomena ${ }^{7,8}$}

If the lattice vibrations are independent of the configurations of the atoms on the lattice sites, we may split the partition function into a vibrational factor and a configurational factor. In this case, the thermodynamic functions of the system are given by the sum of the corresponding functions evaluated separately for the two factors. By way of introduction, let us consider an $\mathrm{AB}_{x}$ alloy, the disorder of which is capable of being described in terms of two interpenetrating sublattices $(\alpha$ and $\beta$ ). The configurational factor of the partition function may be written

$$
Z(T, S)=\sum_{m} \exp \left(-W_{m} / k T\right)
$$

where $T$ denotes the absolute temperature, $S$ is a suitably defined parameter specifying the state of order of the alloy, and $k$ is the Boltzmann constant. $W_{m}$ represents the configurational energy of the state $m$, with the summation extending over all states $m$ corresponding to a given order $S$.

The main contribution to $W_{m}$ may be assumed to come from the interaction energy of pairs of nearest neighbors, $\alpha$ and $\beta$. If the interaction energies of $A-A$, $A-B$, and $B-B$ nearest neighbor pairs are denoted by $-v_{\mathrm{AA}},-v_{\mathrm{AB}}$, and $-v_{\mathrm{BB}}$, respectively and the number of such pairs by $n_{\mathrm{AA}}, n_{\mathrm{AB}}$, and $n_{\mathrm{BB}}$, we may regard the total interaction energy as the sum of individual contributions

$$
W_{m}=-\left\{n_{\mathrm{AA}} v_{\mathrm{AA}}+n_{\mathrm{AB}} v_{\mathrm{AB}}+n_{\mathrm{BB}} v_{\mathrm{BB}}\right\} .
$$

The configurational free energy $F(s)$, corresponding

${ }^{7}$ E. W. Elcock, Order-disorder Phenomena (Methuen and Co., Ltd., London, 1956), Chaps. 2, 4.

${ }_{8}$ T. Muto and Y. Takagi, Solid State Physics, F. Seitz and D. Turnbull, Eds. (Academic Press Inc., London, 1955), Vol. 1, pp. 207-220. to a given $S$, is

$$
F(s)=-k T \ln Z(T, S),
$$

the minimizing of which gives the equilibrium value of $S$ at a given temperature. The corresponding energy of the alloy is

$$
\begin{aligned}
E(S) & =\langle W(S)\rangle \\
& =\sum_{m} W_{m} \exp \left(-W_{m} / k T\right) / \sum_{m} \exp \left(-W_{m} / k T\right) .
\end{aligned}
$$

It is convenient to define $U(S)$ by the relation

$$
\sum_{m} \exp \left(-W_{m} / k T\right)=g(s) \exp (-U(S) / k T),
$$

where the quantity $g(S)$ denotes the total number of possible rearrangements of $\mathrm{A}$ and $\mathrm{B}$ atoms over each sublattice, for a given value of $S$. Whence we have

$$
F(S)=U(S)-k T \ln g(s)
$$

and, from (3),

$$
E(S)=\frac{\partial}{\partial(1 / T)}\left(\frac{U(S)}{T}\right)
$$

We have set up all the necessary equations for treating the order-disorder transition, but it still remains to evaluate the partition function, from which the equilibrium value of $S$, the most probable configurational energy, and the corresponding specific heat may all be obtained. In order to do this, we shall use the simplest method of approximation-the zerothorder, or Bragg-Williams approximation.

In this approximation, the true value of $W_{m}$ for each configuration of a given $S$ is replaced by the simple average $\left\langle W_{m}\right\rangle$ for all states belonging to $S$,

$$
\left\langle W_{m}\right\rangle=-\left\{\left\langle n_{\mathrm{AA}}\right\rangle v_{\mathrm{AA}}+\left\langle n_{\mathrm{AB}}\right\rangle v_{\mathrm{AB}}+\left\langle n_{\mathrm{BB}}\right\rangle v_{\mathrm{BB}}\right\},
$$

where $\left\langle n_{\mathrm{AA}}\right\rangle, \cdots$ are the corresponding averages of $n_{\mathrm{AA}}, \cdots$. We shall always find that $\left\langle W_{m}\right\rangle$, for a given $S$, is independent of temperature. Then we have, from (5),

$$
U(S)=E(S)=\left\langle W_{m}\right\rangle,
$$

as far as our approximation is valid. The configurational specific heat is then

$$
C_{v}{ }^{\text {config }}=(\partial / \partial T)\left\{\left\langle W_{m}\right\rangle\right\} .
$$

\section{Cooperative Disorder of $\mathrm{Ag}^{+}$in $\alpha-\mathrm{AgI}$}

Let us now consider the behavior of the silver ions in $\alpha$-AgI. These ions may be regarded as being co-operatively disordered over a lattice determined by the (b) sites. The (d) and (h) sites clustered about each (b) site ensure the equivalence of vibrational states for all configurations of the ions, while being adequately included in the theory by considering the cluster as a 


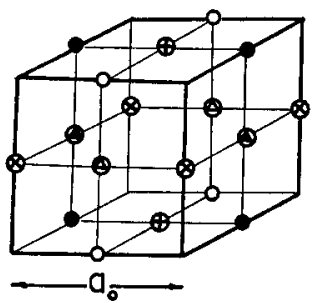

Fig. 3. The six equivalent sublattices available to silver ions. Each symbol represents a cluster of potential wells.

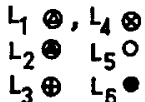

rather complicated potential well centered on the (b) site. This implies that the (b) sites have lowest energy, an assumption which seems reasonable. We shall first consider the configurational factor of the partition function.

The "alloy," $\mathrm{AB}_{2}$ in this case, comprises silver ions (designated A) and silver ion vacancies (designated B) distributed over the lattice shown in Fig. 3. This lattice may be divided into six interpenetrating simple cubic sublattices $L_{i}(i=1,2 \cdots, 6)$, each having the lattice constant $a_{0}$. The convention for numbering these sublattices is also shown in Fig. 3.

For this system, we must determine the total average configurational energy as a sum of energies like that in (6), but referring as well to all possible pairs of sublattices $L_{i}$ and $L_{j}$. If we denote the averages corresponding to $\left\langle n_{\mathrm{AA}}\right\rangle, \cdots$ by $n_{\mathrm{AA}}{ }^{i j}, \cdots$ and the interaction energies by $-v_{\mathrm{AA}}{ }^{i j}, \cdots$, we may rewrite Eq. (6) in the form

$$
\begin{aligned}
\left\langle W_{m}\right\rangle & \equiv \sum_{i, j>i}\left\langle W_{m}\right\rangle^{i j} \\
& =-\sum_{i, j>i}\left\{n_{\mathrm{AA}} i j_{v_{\mathrm{AA}}} i j+n_{\mathrm{AB}}{ }^{i j_{V_{\mathrm{AB}}}}{ }^{i j}+n_{\mathrm{BB}}{ }^{i j_{V_{\mathrm{BB}}}}{ }^{i j}\right\} .
\end{aligned}
$$

We shall specify the state of order of the sublattice $L_{i}$ by the parameter $s_{i}$, the probability that a site on the $i$ th sublattice will be occupied by an atom of type A. Considering a crystal containing $\mathfrak{N}$ sites of each sublattice, we see that the average numbers of $A$ and $\mathrm{B}$ atoms on the sublattice $L_{i}$ are $\Re s_{i}$ and $\Re\left(1-s_{i}\right)$, respectively. Each site on $L_{i}$ is surrounded by $\boldsymbol{z}^{i j}$ nearest neighbor sites of $L_{j}(i \neq j)$, of which the average fractional occupations by $\mathrm{A}$ and $\mathrm{B}$ atoms are $s_{j}$ and $\left(1-s_{j}\right)$, respectively. It follows that

$$
\begin{aligned}
& n_{\mathrm{AA}}{ }^{i j}=\mathscr{N} z^{i j} s_{i} s_{j}, \\
& n_{\mathrm{BB}}{ }^{i j}=\mathfrak{N} z^{i j}\left(1-s_{i}\right)\left(1-s_{j}\right), \\
& n_{\mathrm{AB}}{ }^{i j}=\mathfrak{N} z^{i j}\left\{s_{i}\left(1-s_{j}\right)+s_{j}\left(1-s_{i}\right)\right\} .
\end{aligned}
$$

Following Shockley, ${ }^{9}$ we shall overcome the considerable mathematical complexity experienced after

\footnotetext{
'W. Shockley, J. Chem. Phys. 6, 130 (1938).
}

substituting from Eq. (10) into Eq. (9) by changing the zero point of configurational energy in order to write

where

$$
\left\langle W_{m}\right\rangle^{i j}=-v^{i j} n_{\mathrm{AB}}{ }^{i j},
$$

$$
v^{i j} \equiv v_{\mathrm{AB}}{ }^{i j}-\frac{1}{2}\left(v_{\mathrm{AA}}^{i j}+v_{\mathrm{BB}}{ }^{i j}\right)
$$

is the "ordering energy" between lattices $L_{i}$ and $L_{j}$. Note that at least some of the $v^{i j}$ must be positive quantities if there is to be any ordered state at low temperatures. $^{8}$

Equation (11) may be obtained from Eq. (9) by changing the zero of the configurational energy $\left\langle W_{m}\right\rangle$ by an amount

$$
\begin{aligned}
\delta\left\langle W_{m}\right\rangle= & \sum_{i, j>i}\left\{\frac { 1 } { 2 } \left[v_{\mathrm{AA}}{ }^{i j}\left(2 n_{\mathrm{AA}}{ }^{i j}+n_{\mathrm{AB}}{ }^{i j}\right)\right.\right. \\
& \left.\left.+v_{\mathrm{BB}}{ }^{i j}\left(2 n_{\mathrm{BB}^{i j}}+n_{\mathrm{AB}}{ }^{i j}\right)\right]\right\} \\
= & \sum_{i, \gg>i}\left\{\frac{1}{2} z^{i j}\left[v_{\mathrm{AA}}{ }^{i j} N_{\mathrm{A}}^{i j}\left(s_{i}, s_{j}\right)+v_{\mathrm{BB}}^{i j} N_{\mathrm{B}}^{i j}\left(s_{i}, s_{j}\right)\right]\right\},
\end{aligned}
$$

where $N_{\mathrm{A}}^{i j}\left(s_{i}, s_{j}\right)=\left(2 / z^{i j}\right) n_{\mathrm{AA}}{ }^{i j}+\left(1 / z^{i j}\right) n_{\mathrm{AB}}{ }^{i j}$ is the number of $\mathrm{A}$ atoms distributed over $L_{i}$ and $L_{j}$, under the orderings $s_{i}$ and $s_{j}$.

For our particular lattice,

$$
z^{14}=z^{15}=z^{24}=z^{26}=z^{35}=z^{36}=2,
$$

while the energies $v_{\mathrm{AA}}{ }^{14}, \cdots$ are those corresponding to interaction over the separation, $a_{0} / 2$, of the nearest neighbors. Similarly,

$$
z^{12}=z^{13}=z^{23}=z^{45}=z^{56}=z^{46}=4
$$

with nearest neighbor separation $a_{0} \sqrt{2} / 2$, and

$$
z^{16}=z^{25}=z^{34}=8
$$

with separation of nearest neighbors being $a_{0} \sqrt{3} / 2$.

Apparently insuperable algebraic complexity is encountered unless it can be assumed that the quantities $z^{i j} v_{\mathrm{AA}}{ }^{i j}, \cdots$ are independent of the particular sublattices $i$ and $j$ involved. This will be the case for the present structure if the interaction potentials vary as $-1,-\frac{1}{2},-\frac{1}{4}$, for the three distances $a_{0} / 2, a_{0} \sqrt{2} / 2$, $a_{0} \sqrt{3} / 2$. Such a variation, having a fall-off rather sharper than a shielded Coulombic potential, is not physically unreasonable and since interactions with more distant neighbors have been neglected above, we shall make this assumption in the spirit of our zeroth-order approximation.

Writing $z^{i j_{v_{\mathrm{AA}}}}{ }^{i j}, \cdots$ as $\omega_{\mathrm{AA}}, \cdots$ and noting that $N_{\mathrm{A}}{ }^{i j}\left(s_{i}, s_{j}\right)$ is the sum of the numbers of $\mathrm{A}$ atoms on each sublattice separately, $\left[N_{\mathrm{A}}^{i}\left(s_{i}\right)+N_{\mathrm{A}}^{j}\left(s_{j}\right)\right]$, Eq. (13) becomes

$$
\begin{aligned}
\delta\left\langle W_{m}\right\rangle & =\frac{1}{2} \omega_{\mathrm{AA}} \sum_{i, j>i}\left(N_{\mathrm{A}}^{i}+N_{\mathrm{A}}^{j}\right)+\frac{1}{2} \omega_{\mathrm{BB}} \sum_{i, j>i}\left(N_{\mathrm{B}}^{i}+N_{\mathrm{B}}^{j}\right) \\
& =\frac{5}{2}\left\{\omega_{\mathrm{AA}} N_{\mathrm{A}}^{\text {total }}+\omega_{\mathrm{BB}} N_{\mathrm{B}}^{\text {total }}\right\} .
\end{aligned}
$$


For an alloy of fixed fractional composition $n_{\mathrm{A}}, n_{\mathrm{B}}$ (where $n_{\mathrm{A}}+n_{\mathrm{B}}=1$ ) the shift of the energy zero is then constant. For our purposes, therefore, the changed energy scale will not necessitate corrections to the configurational specific heat.

Substituting from Eq. (10) into Eq. (11), we obtain

$$
\begin{aligned}
\left\langle W_{m}\right\rangle & =-\Re \omega \sum_{i, j>i}\left\{s_{i}\left(1-s_{j}\right)+s_{j}\left(1-s_{i}\right)\right\} \\
& =-\Re \omega \sum_{i, j \neq i}\left\{s_{i}\left(1-s_{j}\right)\right\},
\end{aligned}
$$

where

$$
\omega \equiv z^{i j_{y} i j}=\text { constant. }
$$

Clearly, the number of configurations with a given set of values of the $s_{i}$ is

$$
g(s)=\prod_{i}\left\{\Re ! /\left(\Re s_{i}\right) !\left[\Re\left(1-s_{i}\right)\right] !\right\}
$$

Applying Stirling's approximation in the form $\ln N ! \sim$ $N \ln N$, and using Eqs. (14), (7), (8), and (4), we find that

$$
\begin{array}{r}
F\left(s_{i}\right)=\Re k T \sum_{i}\left\{s_{i} \ln s_{i}+\left(1-s_{i}\right) \ln \left(1-s_{i}\right)\right\} \\
-\Re \omega \sum_{i, j \neq i}\left\{s_{i}\left(1-s_{j}\right)\right\}
\end{array}
$$

and

$$
C_{\nu}{ }^{\operatorname{config}}(T)=-\mathfrak{N} \omega(\partial / \partial T) \sum_{i, j \neq i}\left\{s_{i}\left(1-s_{j}\right)\right\} .
$$

The equilibrium values of $s_{i}$ are found by minimizing the free energy $F\left(s_{i}\right)$ subject to the condition

$$
\sum_{i} s_{i}=6 n_{\mathrm{A}}=\text { constant }
$$

which expresses the requirement that the composition should remain fixed. This is conveniently accomplished by using the method of Lagrange multipliers when, introducing the undetermined multiplier $\beta$, we maximize

$$
\ln Z\left(s_{i}\right)+\beta \sum_{i} s_{i}
$$

treating the $s_{i}$ as independent variables. Performing the partial differentiations and using (18), we obtain

FIG. 4. Three possible solutions of the equation $f(s)=\lambda$ for the particular temperature $\tau=0.7$ (after Shockley ${ }^{9}$ ). The form of the curve does not change radically with temperature.

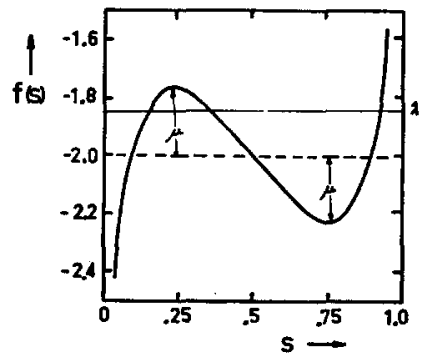

FIG. 5. Ordering parameters $\sigma$ and $S$ as functions of reduced temperature $\tau$. The solution having the larger value of $\sigma$ at a given $\tau$ represents the equilibrium state.

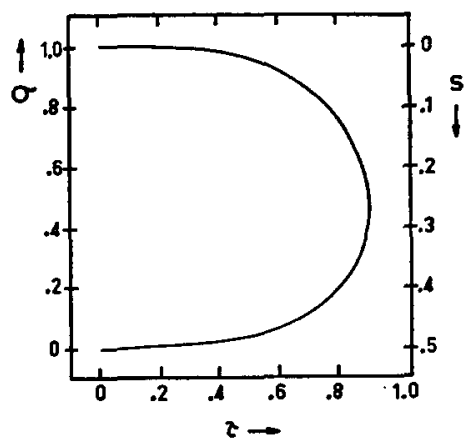

the simultaneous equations

$$
f\left(s_{i}\right) \equiv-4 s_{i}+\tau \ln \left[s_{i} /\left(1-s_{i}\right)\right]=\lambda,
$$

where $\tau$ is the "reduced temperature",

$$
\tau=2 k T / \omega
$$

and $\lambda=2\left(5-12 n_{\mathrm{A}}\right)+\tau \beta / \Re$ may be regarded as an undetermined parameter.

Figure 4 shows the behavior of the left-hand side of Eq. (19) as a function of $s_{i}$ for a particular temperature $(\tau=0.7) .^{9}$ For $-2+\mu>\lambda>-2-\mu$, where $\mu$ is defined in the figure, there are three roots of Eq. (19). These roots are to be assigned to the six order parameters $s_{i}$ in some way, each way of assigning the roots corresponding to a given kind of ordering. For a general alloy, there is no simple way of telling which values should be assigned to which parameters, it being necessary to use Eq. (16) to determine which set of values has the lowest free energy for a given temperature and composition. For $\lambda$ outside this range there is only one real value of $s_{i}$ and it is not generally possible to find a physically acceptable solution.

In the case of $\alpha$-AgI, however, the appropriate kind of ordering is determined primarily by the iodide ions and not solely by the kinetics of the $\mathrm{AB}_{2}$ "alloy" describing the distribution of silver ions. We have already seen that ordering of the silver ions will necessarily cause some distortion of the body-centered lattice of iodide ions. It seems reasonable to assume that the most probable ordered structure corresponds to a body-centered tetragonal lattice of iodide ions wherein the lattice spacing has been increased in one direction only. The corresponding ordered structure of silver ions is obtained by assigning the occupation probability unity to two of the sublattices and zero to the other four, the two occupied sublattices being either $L_{1}$ and $L_{6}, L_{2}$ and $L_{5}$, or $L_{3}$ and $L_{4}$. We shall therefore assume, for reasons of symmetry and continuity, that the silver ions disorder in such a way that four of the parameters $s_{i}$ have the same value, $S$, and the other two have the value $\sigma$. From Eq. (18) with $n_{\mathrm{A}}=\frac{1}{3}$, we see that there is now only one independent order parameter since

$$
2 S+\sigma=1
$$




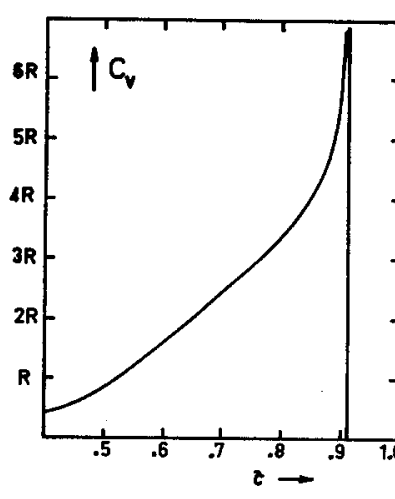

Fig. 6. Configurational specific heat of silverions as a function of reduced temperature $\tau$.

and the undetermined multiplier in Eq. (19) may be eliminated, giving

$$
\tau=2(3 \sigma-1) / \ln \left(\frac{\sigma}{1-\sigma} \frac{1+\sigma}{1-\sigma}\right)
$$

from which $r$ is readily found as a function of $\sigma$. We see from Fig. 5 that Eq. (22) predicts two values of $\sigma$ at any given temperature. This occurs because we have actually found the values of $\sigma$ for which the free energy is stationary. In this case, the larger value of $\sigma$ represents the equilibrium value at a given temperature. As the temperature is increased from zero, where the stable state is given by $\sigma=1$ and $S=0$, the difference between the two values of $\sigma$ decreases until a temperature $T_{m}$ is reached at which they are equal. The temperature $T_{m}$ represents the critical temperature for the "alloy" which is completely disordered at all temperatures greater than $T_{m}$.

Using Eq. (18), we may write the differential in Eq. (17) as

$$
\begin{aligned}
(\partial / \partial T)\left[\sum_{i, j \neq i}\right. & \left.s_{i}\left(1-s_{j}\right)\right] \\
& \equiv(\partial / \partial T)\left\{\sum_{i} s_{i} \sum_{j}\left(1-s_{j}\right)-\sum_{i} s_{i}\left(1-s_{i}\right)\right\} \\
& =(\partial / \partial T)\left\{\text { constant }-\sum_{i} s_{i}\left(1-s_{i}\right)\right\}
\end{aligned}
$$

and use Eq. (20) to obtain

$$
C_{v}{ }^{\operatorname{config}}(T)=2 \Re k(\partial / \partial \tau)\left\{\sum_{i} s_{i}\left(1-s_{i}\right)\right\} .
$$

Now $n_{\mathrm{A}}=\frac{1}{3}$, so we need 29 silver ions to occupy the $6 \mathfrak{N}$ lattice sites of the alloy. Putting $2 \Re=N$, Avogadro's number, we see that the molar configurational specific heat is

$$
\begin{aligned}
C_{v}^{\text {onfig }}(T) & =R(\partial / \partial \tau) \sum_{i} s_{i}\left(1-s_{i}\right) \\
& =R(\partial / \partial \tau)\{(1-\sigma)(1+3 \sigma)\} .
\end{aligned}
$$

Performing the necessary calculations with the results of Fig. 5, we obtain the configurational specific heat shown as a function of reduced temperature in
Fig. 6. As a check of this result, we see that the values of Fig. 6 are roughly the same as those obtained for alloys with the Bragg-Williams approximation, realizing that a factor of three is introduced because one mole of silver ions includes a total of three moles of "atoms" in our particular $\mathrm{AB}_{2}$ system.

Before it is possible to compare this theory with experiment, we need to know the critical temperature $T_{m}$. We have seen ${ }^{1}$ that the results of Lieser ${ }^{10}$ between $180^{\circ}$ and $320^{\circ} \mathrm{C}$ are in agreement with those of the present work, which show a steady increase in the specific heat $c_{p}$ from $18 \mathrm{cal} / \mathrm{mole} \cdot{ }^{\circ} \mathrm{C}$ just above the $\beta$-AgI to $\alpha$-AgI transition temperature $150^{\circ} \mathrm{C}$, to 27 $\mathrm{cal} / \mathrm{mole} \cdot{ }^{\circ} \mathrm{C}$ at $360^{\circ}$, the highest temperature investigated. Lieser also measured $c_{p}$ at $420^{\circ} \mathrm{C}$, obtaining a value of $30.4 \mathrm{cal} / \mathrm{mole} \cdot{ }^{\circ} \mathrm{C}$ which suggests that there is a rapid rise in specific heat near $400^{\circ} \mathrm{C}$. It would seem, therefore, that the critical temperature lies between $400^{\circ}$ and $555^{\circ} \mathrm{C}$, the melting temperature. For the purpose of comparison, we will set the critical temperature equal to the melting temperature, noting that if $T_{m}$ is lower than this the predicted heat capacity will be increased in the range of measurement. A calorimeter is being constructed with a view to making a careful investigation of the temperature range $400^{\circ}-$ $600^{\circ} \mathrm{C}$.

\section{Vibrational Specific Heat of Silver Ions in $\alpha-\mathrm{AgI}$}

Our next task is to determine the specific heat contributed by the vibrational factor of the partition function. We have already made the assumption that the (b) sites have lower potential energy than the (d) and (h) sites clustered about them. The average number of sites in each cluster is four (h) sites and two (d) sites, because each of the four (d) sites around a given (b) site is effectively shared between two (b) sites. Considering a given cluster then, we see that the potential $V(x, y, z)$ may be roughly separated into two parts, $V(x, y)$ and $V(z)$, where the $(x, y)$ plane is taken as the plane of the cluster. The part $V(z)$ is to a first approximation a harmonic potential, while $V(x, y)$ must include the complicated nature of the cluster. The potential required for $V(x, y)$ must include a deep central well [the (b) site] with six subsidiary wells of higher potential positioned about it. Four of these wells must be at the same potential, representing the (h) sites, and the other two should be at some other height representing the (d) sites.

The calculations are simplified considerably if we assume a potential $V(x, y, z)$ which is completely separable in Cartesian coordinates. In this case, the total specific heat is the sum of the specific heats corresponding to the three one-dimensional potentials. An appropriate vibrational potential for the cluster is

$$
V(x, y, z)=V(x)+V(y)+V(z),
$$

${ }^{10}$ K. H. Lieser, Z. Physik. Chem. (Frankfurt) 2, 238 (1954). 
where

$$
\begin{aligned}
V(z) & =E_{z} z^{2} / b^{2}, & & \\
V(x) & =\epsilon_{x}+\left(E_{x} / a^{2}\right)(x+2 a)^{2}, & & \text { for } x \leq-a \\
& =\left(E_{x}+\epsilon_{x}\right) x^{2} / a^{2}, & & \text { for }-a \leq x \leq a \\
& =\epsilon_{x}+\left(E_{x} / a^{2}\right)(x-2 a)^{2}, & & \text { for } x \geq a,
\end{aligned}
$$

and $V(y)$ is of the same form as $V(x)$, but with parameters $\epsilon_{y}$ and $E_{y}$. The potentials $V(x)$ and $V(y)$ have the form of a central well, flanked by two shallower wells as shown in Fig. 7. The total potential has a well with base level zero at the point $(0,0,0)$ representing the (b) site, two wells at $( \pm 2 a, 0,0)$ with base $\epsilon_{x}$ representing (d) sites, and two wells at $(0, \pm 2 a, 0)$ with base $\epsilon_{y}$ representing (h) sites. In the calculation each of these last two wells is given a statistical weight of 2 in order to allow for the total of four $(h)$ sites in the cluster. The total potential also has supernumery wells at $( \pm 2 a, \pm 2 a, 0)$ which do not occur in the physical situation but their contribution to the specific heat is very small because of their high base level $\epsilon_{x}+\epsilon_{y}$. Calculations with a variety of modifications of this model show that the specific heat is not sensitive to details of positioning and boundary conditions for individual wells, as follows indeed from the nature of the classical partition function, so that this potential should give a reasonable approximation to the physical situation.

At the temperature of the experiment, the ions may be regarded as being classical particles and their partition function and vibrational specific heat may be calculated with comparative ease. The results of these calculations for a one-dimensional potential of the form $V(x)$, but with side wells having degeneracy $n$, is shown in Fig. 7 for various relationships between $\epsilon$ and $E$, and also for the two cases $n=1$ and $n=2$ which represent the potentials $V(x)$ and $V(y)$, respectively. The vibrational specific heat of a mole of silver ions is obtained by adding the appropriate ordinates of the two sets of curves in Fig. 7 and the contribution, $R$, from $V(z)$.

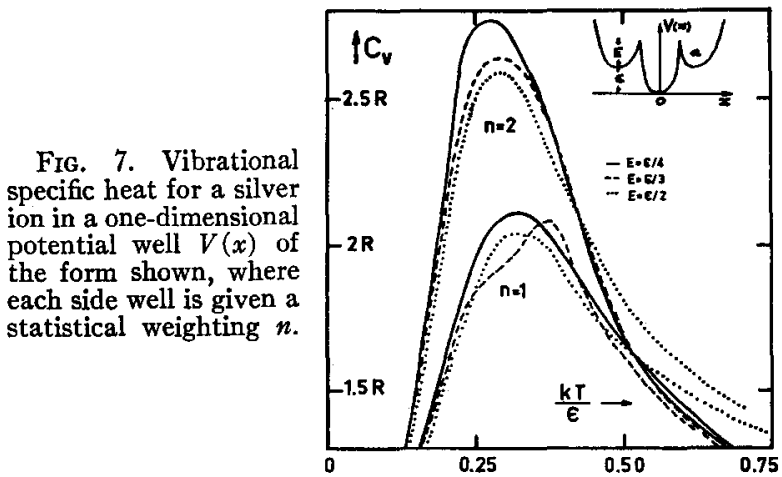

In order to get the best possible agreement with experiment we need to attribute as much specific heat as possible to these vibrational modes over the temperature range $150^{\circ}-555^{\circ} \mathrm{C}$. There is little experimental information about the base levels of the individual wells in the potential and our choice is constrained only by the necessity not to conflict with $x$-ray structure data and ionic conductivity measurements. We have chosen to take the potentials of sites (d) and (h) to be equal and $3.6 \mathrm{kcal} / \mathrm{mole}$ above that of the (b) sites.

\section{Specific Heat of the Iodide Ions in $\alpha$-AgI}

In the ordered state, as we have seen, the iodide ions may be assumed to occupy a body-centered tetragonal lattice, while two of the six sublattices for silver ions are occupied. Disorder of the silver ions occurs by the movement of the ions off these two lattices, to become distributed over the other four sublattices. When a given silver ion moves in this way, two iodide ions must move apart to accommodate it, while the two iodide ions nearest the vacancy created by this shift of the silver ion will move closer together. For a mole of $\alpha$-AgI in a state of disorder characterized by the parameter $S$ of Eq. (21), there are $2 N S$ silver ions which have moved in this way. The corresponding positions of the iodide ions are only uniquely determined if all distortions of their lattice are symmetrical, that is if a given pair involved in the movement instigated by a silver ion move apart or together in such a way that each is displaced by the same distance. In this case, there is no uncertainty in the positions of the iodide ions and no configurational specific heat.

It is possible, however, that one ion of a pair may be displaced more than the other. The entropy associated with the uncertainty as to which ion is displaced gives rise to a configurational specific heat for the iodide ions. In order to estimate the magnitude of this specific heat contribution arising from disorder among the iodide ions, we observe that if there are $2 N S$ silver ions displaced from their ordered positions then, neglecting interactions between them, this leads to $4^{2 N S}$ possible iodide ion configurations and a configurational entropy contribution of $2 N k S \ln 4$. The corresponding contribution to the specific heat is negligible below $250^{\circ} \mathrm{C}, 1.4 \mathrm{cal} / \mathrm{mole} \cdot{ }^{\circ} \mathrm{C}$ at $350^{\circ} \mathrm{C}$ and $2.8 \mathrm{cal} / \mathrm{mole} \cdot{ }^{\circ} \mathrm{C}$ at $450^{\circ} \mathrm{C}$. A more careful calculation would give a smaller value, particularly above $450^{\circ} \mathrm{C}$ where this simple calculation overestimates the number of configurations and the predicted specific heat contribution rises steeply to greater than $6 \mathrm{cal} / \mathrm{mole} \cdot{ }^{\circ} \mathrm{C}$ at $540^{\circ} \mathrm{C}$.

In addition to the specific heat contributed by their disorder, the $N$ iodide ions have a vibrational specific heat $3 R$ obtained by assuming that each ion vibrates in a harmonic potential well whose position in space is determined by the distribution of silver ions. 


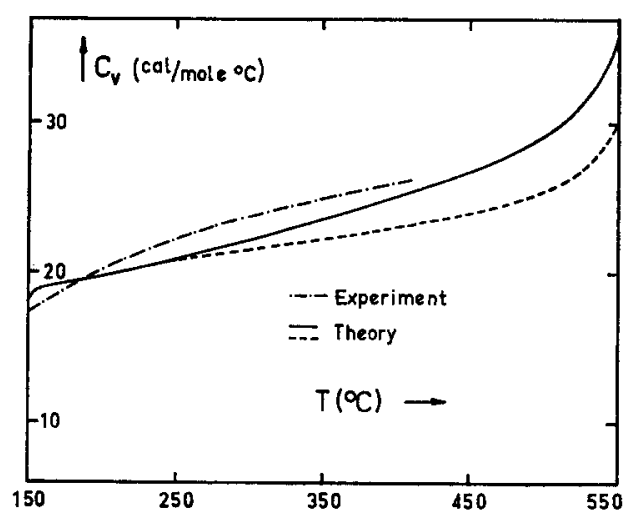

Fig. 8. Comparison of calculated heat capacity for $\alpha$-AgI with experiment. The full curve includes the calculated disorder contribution from iodide ions while the broken curve omits this term.

\section{CONCLUSIONS}

\section{Stoichiometric Silver Iodide}

The molar heat capacity of $\alpha$-AgI is obtained by adding the corresponding values of the configurational and vibrational heat capacities of both silver and iodide ions. The result shown in Fig. 8 relies on the assumption that the critical temperature for the cooperative disordering of silver ions is actually the melting point of $\alpha-\mathrm{AgI}, 555^{\circ} \mathrm{C}$. Two theoretical curves are shown in Fig. 8; the lower curve corresponds to a crystal wherein there is no configurational specific heat contributed by the iodide ions, while the other curve includes the contribution estimated immediately above. The true molar heat capacity predicted by our model lies somewhere between the two and is seen to be in quite good agreement with the experimental curve.

Since the model involves a number of assumptions for which there is no direct experimental evidence, experiments are at present being planned to test some of these assumptions. The most important of these will involve the use of $\mathrm{x}$-ray diffraction techniques to investigate ordering in large-grain polycrystalline samples of $\alpha$-AgI.

\section{Nonstoichiometric Silver Iodide}

We have seen ${ }^{1}$ that samples of silver iodide which deviate from stoichiometry by as little as 1 mole $\%$ have a heat capacity which is markedly different from that of stoichiometric samples (Fig. 1). This behavior may be partially explained if we assume that the lack of stoichiometry induces essentially complete disorder at any temperature above the $150^{\circ} \mathrm{C}$ phase transition. The parameter $\sigma$ would then be independent of temperature and there would be no configurational specific heat for either ionic species. Attributing a vibrational specific heat $3 R$ to the iodide ions, we must assume also, in order to explain the observed specific heat values, that some effect enters to reduce the vibrational specific heat of the silver ions to the same value. This would occur if the difference in base energy between (b) sites and either (d) or (h) sites for silver ions in disordered positions became close to $k T$, instead of about $3 k T$ as assumed for partially ordered material. If this level shift were to occur it would not cause a great change in the calculated heat capacity curves for stoichiometric material, except quite close to the melting point, and would lead to a specific heat for nonstoichiometric material actually decreasing with rising temperature, as is in fact observed.

The electrical conductivity data of Tubandt and Loren $z^{11}$ lend some support to this interpretation. This data was obtained with reversible silver electrodes and is consequently applicable to silver iodide in equilibrium with metallic silver. The ionic conductivity was observed to increase slowly with temperature, reaching a value at the melting point higher than the conductivity of the melt. This behavior was attributed to a structure in which the silver ions are essentially liquid and in which the activation energy for their migration is very small-exactly the model proposed here for the nonstoichiometric material.

We propose to perform controlled measurements of ionic conductivity using inert electrodes which should give important information about the proposed models for stoichiometric and nonstoichiometric alpha silver iodide, because these models predict very different temperature dependence of the ionic conductivity. For our model of stoichiometric $\alpha$-AgI, we may show that a silver ion moving from its ordered position to a neighboring disordered position causes an increase in configurational energy which is proportional to $(\sigma-S)$. Although the barrier to silver-ion migration depends on the microscopic detail of the interaction potentials, it should also be proportional to $(\sigma-S)$, therefore decreasing with increasing temperature, and causing a sharply rising ionic conductivity.

In Paper III of this series, we will report calorimetric investigations with quenched and slowly cooled samples of both stoichiometric and nonstoichiometric silver iodide. It will be seen that the experimental evidence therein gives support to the proposed models and indicates the existence of a critical temperature for disordering of stoichiometric AgI at approximately $430^{\circ} \mathrm{C}$.

\section{ACKNOWLEDGMENTS}

This work is part of a program of study of partially disordered solids supported by the University of New England and by the Australian Research Grants Committee. One of us (C.M.P.) is also grateful to the Commonwealth Scientific and Industrial Research Organization for a scholarship, during the tenure of which this work was done.

\footnotetext{
11 C. Tubandt and E. Lorenz, Z. Physik. Chem. 87, 513, 543 (1914)
} 